\title{
Improvements in EPMA: Spatial Resolution and Analytical Accuracy
}

\author{
P. K. Carpenter ${ }^{1}$ and B. L. Jolliff ${ }^{2}$ \\ ${ }^{1}$ Dept. of Earth and Planetary Sciences, Washington University, St. Louis, MO 63130, USA \\ ${ }^{2}$ Dept. of Earth and Planetary Sciences and the McDonnell Center for the Space Sciences, Washington \\ University, St. Louis, MO 63130, USA
}

In electron-probe microanalysis (EPMA), the analyst must balance the desire for high spatial resolution with practical aspects of quantitative analysis. A reduction in accelerating voltage and probe current improves spatial resolution and image quality, but forces the selection of X-ray lines with low excitation energy (e.g., L and M-family X-ray lines vs. K and L-family lines). Measurement of L-family X-rays is complicated by low fluorescent yield, an increase in X-ray absorption, and numerous X-ray interferences from other elements in the sample. The use of transition element L $\alpha$ lines generally results in both poor sensitivity and accuracy.

Lunar agglutinates are rock and mineral soil materials included in glass formed by micrometeorite impact events and exposure to solar wind processes [1]. Agglutinates contain sub-micron spherical droplets of $\mathrm{Fe}$ metal that form initially on the surface of grains during exposure to energetic particles. Impact processes rework the regolith, resulting in agglutinates containing mineral and rock fragments with melt and quench textures and pervasive metal spherules. Micron sized spherules are variably derived from melted minerals plus a meteoritic component, and may contain $\mathrm{Ni}, \mathrm{P}$, and $\mathrm{Co}$, but nanophase $\mathrm{Fe}$ is essentially pure $\mathrm{Fe}$, and the relationship between spherule size and chemistry has not been extensively studied. The fine scale of these spherules requires microanalysis at high spatial resolution as well as consideration of sampling, measurement, and accuracy. An Apollo 17 agglutinate 76503,7020 from the study by Jolliff et. al. [2] has been selected to evaluate spatial resolution and accuracy of EPMA. Gopon et. al. have analyzed submicron Fe-Si droplets from Apollo 16 soils at low voltage, using the Fe Ll X-ray line rather than the Fe La line due to accuracy issues with the latter [3]. The chemistry of Fe spherules is an indicator of oxygen fugacity and source material, and thus provides insight into both the conditions and source materials which form agglutinates. The current study will present aspects of microanalysis and the relative merits of multiple $\mathrm{kV}$, reduced $\mathrm{kV}$, and low-kV measurement methodology to improve spatial sampling coupled with an assessment of analytical accuracy.

Agglutinate 76503,7020 consists of glass with inclusions of regolith materials such as rock fragments and mineral grains with textures indicative of impact melting events (Fig. 1). Submicron Fe spherules are pervasive and abundant at grain boundaries and FeTi-rich zones in the agglutinate glass. Preliminary EPMA of micron-sized $\mathrm{Fe}$ spherules indicates that they contain minor $\mathrm{Ni}$ and $\mathrm{P}$ with trace $\mathrm{Co}$, thus suggesting a meteoritic contribution. The fundamental problem with microanalysis of the spherules is to discriminate between elements in the spherule vs. elements in the matrix. It is necessary to quantitatively determine the electron and X-ray primary analytical volume and degree of sampling of adjacent matrix by secondary fluorescence. Primary electron scattering through the spherule results in preferential excitation of low $\mathrm{Z}$ matrix elements such as $\mathrm{Mg}$ and $\mathrm{Al}$, which are not likely contained in the spherule, but also $\mathrm{Si}$, which may well be. Secondary fluorescence by characteristic and continuum X-rays from spherule elements (e.g., $\mathrm{Fe}, \mathrm{Ni}$, and $\mathrm{P}$ ) may excite $\mathrm{Ca}$ and $\mathrm{Ti}$ (by $\mathrm{Fe} \mathrm{K} \alpha$ and $\mathrm{Ni} \mathrm{K} \alpha$ ) and $\mathrm{Si}$ (by $\mathrm{P} \mathrm{K} \alpha$ ).

Monte Carlo simulations using DTSA-II (version Halley) have been made for a cubic inclusion in a matrix of glass, using EPMA data from larger spherules and adjacent glass [4]. These simulations are a valuable tool to use in combination with calculated electron and X-ray ranges in these materials. The results are shown in Fig. 2 which illustrates a factor of 2 reduction in scattering volume with decrease in accelerating potential from 15 to $10 \mathrm{kV}$. The excitation volume for $\mathrm{Fe}$ and $\mathrm{Ni} \mathrm{K \alpha}$ at $10 \mathrm{kV}$ is modeled to be within a $0.5 \times 0.5 \mu \mathrm{m}$ cube approximating a Fe spherule (Wt.\% element: Fe 96.4, Ni 2.3, P 0.43) embedded in a glass of measured composition. These simulations were run with both characteristic and continuum fluorescence options enabled, which significantly expands the sampled volume. The simulated EDS spectra at $15 \mathrm{vs.} 10 \mathrm{kV}$ show a significant reduction in the matrix elements $\mathrm{Mg}, \mathrm{Al}$, and $\mathrm{Si}$ at $10 \mathrm{kV}$ which indicates these elements are 
present in the matrix glass only. In practice the $\mathrm{Al} / \mathrm{Fe}$ ratio of $\mathrm{Fe}$ spherules is a sensitive indicator of matrix glass sampling by side-scattered electrons.

The accuracy of EPMA at reduced overvoltage has not been well studied. Strong curvature in the X-ray ionization cross-section at overvoltage below $\sim 2$ and poor agreement of algorithms results in the EPMA rule to "avoid overvoltage less than 2" in analytical setups. The excitation efficiency also decreases (e.g., Ni peak intensity at $10 \mathrm{kV}$ in Fig 2), so both replicate analysis and detection limit will suffer at reduced overvoltage. A summary of measurements made on a suite of standards at reduced overvoltage will be discussed to provide a framework for evaluation of the benefits obtained by the improved spatial resolution.

\section{References}

[1] Lucey, P., New Views of the Moon, pp 83-220, Mineralogical Society of America, 2006.

[2] Jolliff et. al., Met. Planet. Sci. 31, 116-145, 1996.

[3] Gopon et. al., Microsc. Microanal. 19, 1698-1708, 2013.

[4] http://www.cstl.nist.gov/div837/837.02/epq/dtsa2/index.html

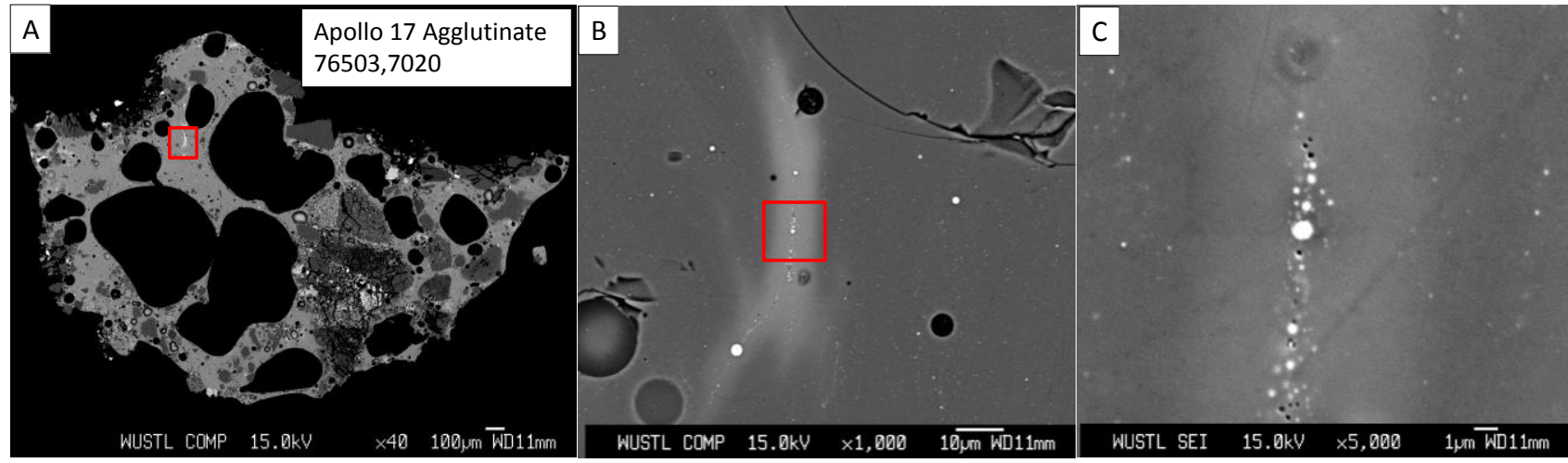

Figure 1 Apollo 17 agglutinate 76503,7020, BSE images of agglutinate and increased magnification of area outlined in red. Fig 1A, Overview image of agglutinate with glass, mineral, and lithic inclusions. Fig 1B, Enlarged area from Fig 1A of FeTi-rich glass and brighter Fe melt spheres. Fig 1C, Enlarged area from Fig 1B illustrating range of nano- and micron-sized Fe melt spheres pervasively distributed in the agglutinate glass.
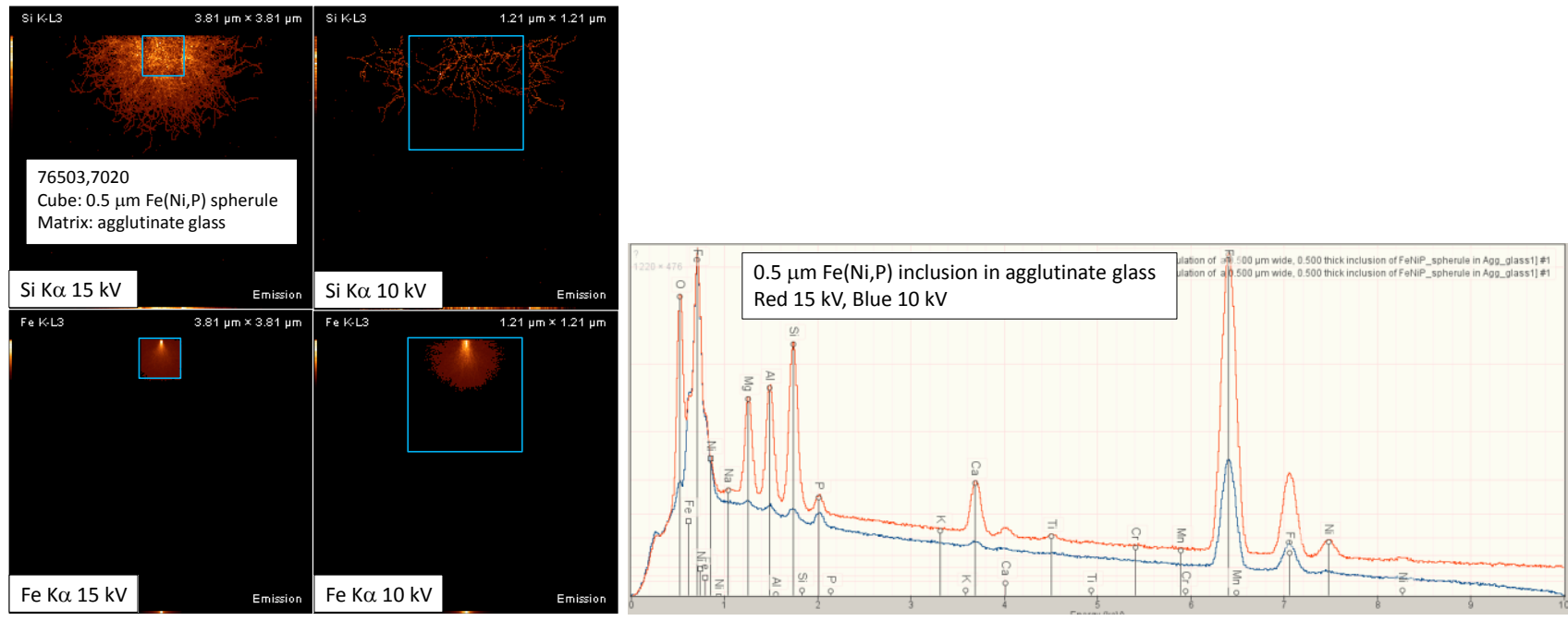

Figure 2 DTSA-II simulations of $0.5 \mu \mathrm{m}$ cube of $\mathrm{Fe}(\mathrm{Ni}, \mathrm{P})$ outlined in blue, in agglutinate matrix glass. Left, Monte Carlo scattering simulation for $\mathrm{Si} \mathrm{K \alpha}$ and $\mathrm{Fe} \mathrm{K \alpha}$ at 15 vs. $10 \mathrm{kV}$. Note $~ 2 \mathrm{x}$ reduction in analytical volume at 10 vs. $15 \mathrm{kV}$. Right, Simulated EDS spectra compared, note significant excitation of matrix elements at $15 \mathrm{kV}$ vs. near elimination of same at $10 \mathrm{kV}$, but reduction in excitation of $\mathrm{Fe}$ and $\mathrm{Ni} \mathrm{K} \alpha$ at $10 \mathrm{kV}$ due to low overvoltage. 